\title{
Haemodynamic Benefit of Cardiac Resynchronisation Therapy Requires Left Bundle Branch Block: A Case Report
}

\author{
M. D. Bogaard • G. E. Leenders • P. A. Doevendans • \\ M. Meine
}

Published online: 5 January 2011

(C) The Author(s) 2010. This article is published with open access at Springerlink.com

\begin{abstract}
A 55-year-old woman with dilated cardiomyopathy and rate-dependent left bundle branch block had a cardiac resynchronisation therapy (CRT) device implanted. During implantation, the maximum rate of left ventricular pressure rise $\left(\mathrm{dP} / \mathrm{dt}_{\max }\right)$ was measured invasively. This case presents a description of the acute negative effect of a left bundle branch block on $\mathrm{dP} / \mathrm{dt}_{\max }$, and the different effect of CRT on left ventricular haemodynamic function in the presence and absence of a left bundle branch block.
\end{abstract}

Keywords Cardiac resynchronisation therapy - Left bundle branch block $\cdot \mathrm{dP} / \mathrm{dt}_{\max }$

\section{Case Report}

The effect of cardiac resynchronisation therapy (CRT) in patients with heart failure without left bundle branch block (LBBB) is disputable [1]. We present a case of a patient with rate-dependent LBBB.

A 55-year-old woman with dilated cardiomyopathy, a left ventricular (LV) ejection fraction of $32 \%$ (bloodpool

\author{
M. D. Bogaard $(\bowtie) \cdot$ G. E. Leenders $\cdot$ P. A. Doevendans $\cdot$ \\ M. Meine \\ Department of Cardiology, University Medical Center Utrecht, \\ Heidelberglaan 100, Q05.2.314, \\ 3584 CX, Utrecht, the Netherlands \\ e-mail: M.D.Bogaard-2@umcutrecht.nl \\ G. E. Leenders \\ e-mail: G.E.H.Leenders@umcutrecht.nl \\ P. A. Doevendans \\ e-mail: P.Doevendans@umcutrecht.nl \\ M. Meine \\ e-mail: M.Meine@umcutrecht.nl
}

radionuclide scintigraphy) and New York Heart Association class III despite optimal pharmacological therapy was referred for CRT device implantation. Coronary angiography revealed no abnormalities. A 24-h electrocardiogram registration showed complete LBBB that disappeared at slower heart rates between 70 and 80 bpm (Fig. 1). Since LBBB was present for the vast majority of the time, the patient was accepted for CRT implantation according to current guidelines [2]. Echocardiography during narrow QRS showed a dilated left ventricle without visual signs of dyssynchrony, normal interventricular mechanical delay $(18 \mathrm{~ms})$ and no LV intraventricular dyssynchrony (septal to lateral strain delay $53 \mathrm{~ms}$ ) or atrioventricular (AV) dyssynchrony.

The patient gave written informed consent for a study that was approved by the institutional ethics committee and complies with the Declaration of Helsinki. During implantation, a pressure sensor tipped wire (PressureWire ${ }^{\circledR 5}$, St. Jude Medical Inc., St. Paul, MN, USA) was placed in the left ventricle to optimise the AV delay by measurement of the maximum rate of LV pressure rise $\left(\mathrm{dP} / \mathrm{dt}_{\max }\right)$. The leads were implanted transvenously in the right ventricular outflow tract, right atrial appendage, and in a coronary sinus tributary on the midposterolateral LV free wall.

Pacing leads, pressure recording, and 12-lead surface electrocardiogram were connected to an external pacing and data acquisition computer (Flexstim II, Boston Scientific Corp., St.Paul, MN, USA). The custom-made stimulation protocol consisted of cycles of six beats of atriobiventricular pacing separated by periods of 14 beats of atrial pacing (AAI, baseline). This cycle was repeated with biventricular pacing without atrial pacing (VDD), separated by periods of no pacing (sinus rhythm, baseline). Each cycle with one of four AV delays $(20 \%, 40 \%, 60 \%$, and 

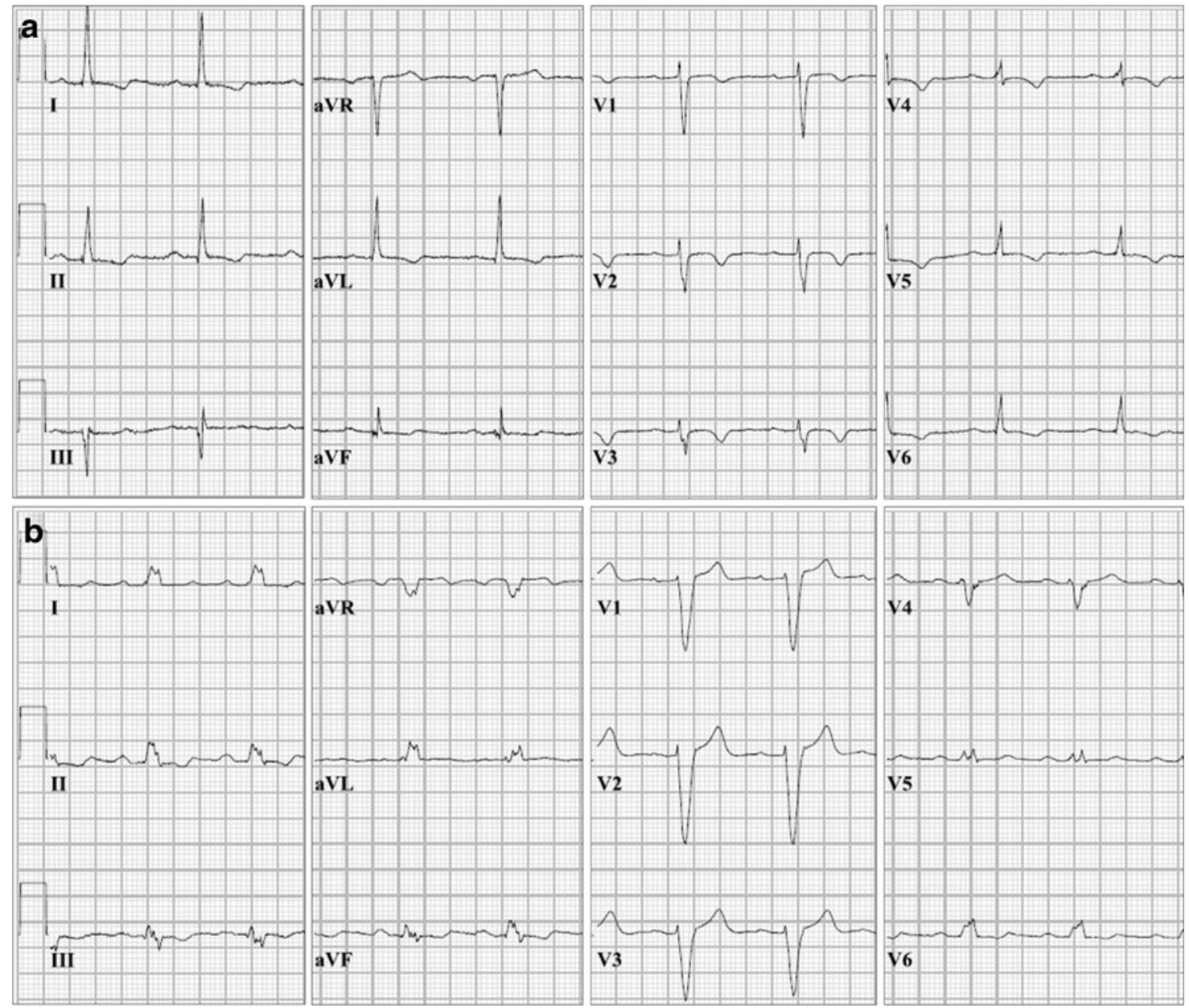

Fig. 1 12-Lead surface electrocardiogram during normal ventricular conduction and during left bundle branch block. a Narrow QRS (90 ms), heart rate $67 \mathrm{bpm}$. b Wide QRS (160 ms) with complete LBBB, heart rate $73 \mathrm{bpm}$

$80 \%$ of intrinsic AV conduction time) was repeated four times in random order. The optimal AV delay was defined by the highest relative increase in $\mathrm{dP} / \mathrm{dt}_{\max }$ compared with baseline.
Complete LBBB was present during AAI pacing at $85 \mathrm{bpm}$ (QRS $160 \mathrm{~ms}$, interventricular delay $130 \mathrm{~ms}$ on intracardiac electrograms). During sinus rhythm (75 bpm) there was no LBBB (QRS $90 \mathrm{~ms}$, interventricular delay
Fig. 2 Acute haemodynamic response to cardiac resynchronisation therapy (CRT) during left bundle branch block (LBBB) and normal ventricular activation. a Absolute $\mathrm{LV} \mathrm{dP/dt}$ max values at baseline (no CRT) and during CRT with four different AV delays. b Relative increase in $\mathrm{LV} \mathrm{dP} / \mathrm{dt}_{\max }$ during CRT compared with baseline without CRT. Mean \pm standard error are shown. Squares narrow QRS, diamond LBBB, AVI intrinsic atrioventricular interval, $A V$ delay atrioventricular delay
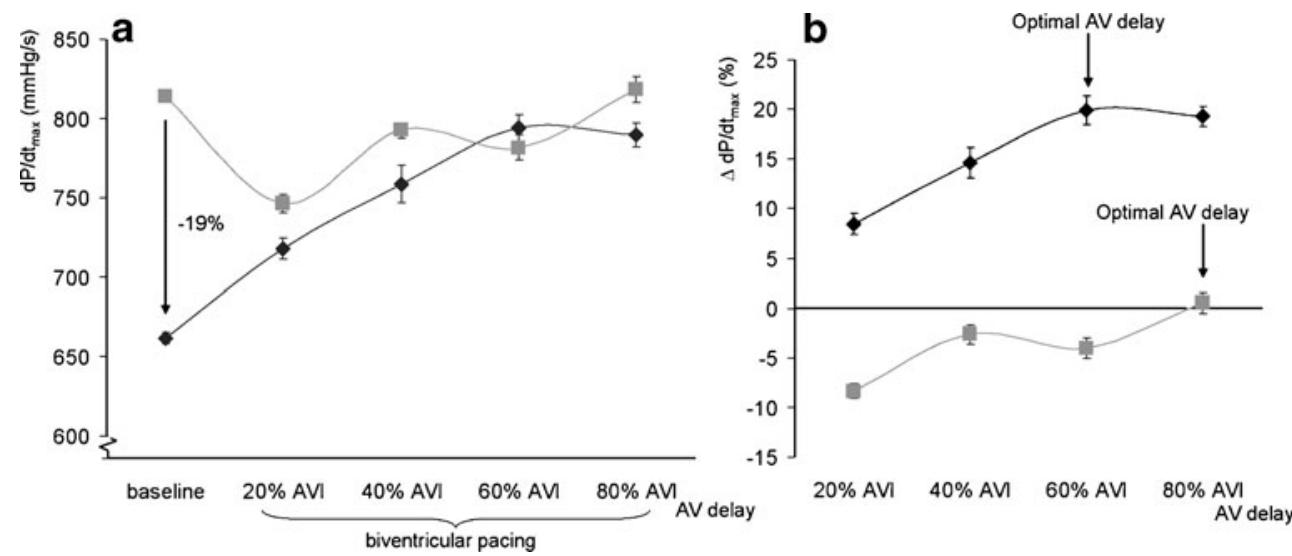
$60 \mathrm{~ms}$ ). LBBB caused a sudden decline in average baseline $\mathrm{dP} / \mathrm{dt}_{\max }$ from $820 \pm 75$ to $662 \pm 52 \mathrm{mmHg} / \mathrm{s} \quad(p<0.001$, paired $t$ test; Fig. 2a). CRT during LBBB increased $\mathrm{dP} /$ $\mathrm{dt}_{\max }$ by $19.9 \%$ (Fig. 2b). In the absence of LBBB, optimised CRT did not change $\mathrm{dP} / \mathrm{dt}_{\max }$ compared with baseline; CRT with short AV delay worsened $\mathrm{dP} / \mathrm{dt}_{\max }$ (Fig. 2b).

\section{Discussion}

$\mathrm{LV} \mathrm{dP} / \mathrm{dt}_{\max }$ is a measure of systolic function that can reflect acute haemodynamic improvement achieved by CRT [3-5]. In this case, AAI pacing induced LBBB and immediately decreased $\mathrm{dP} / \mathrm{dt}_{\max }$. Acute decrease in haemodynamic function by induction of LBBB was previously shown in a preclinical model [6]. Systematic human research about the effect of CRT during both conditions within the same patient is difficult since data before onset of LBBB are often lacking.

In the absence of LBBB, biventricular pacing did not improve $\mathrm{dP} / \mathrm{dt}_{\max }$. In a randomised trial, CRT also failed to improve quality of life and functional and echocardiographic parameters in heart failure patients with QRS $<130 \mathrm{~ms}$ and mechanical dyssynchrony [1]. In this case, CRT with a short AV delay worsened $\mathrm{dP} / \mathrm{dt}_{\max }$, which can be explained by impaired diastolic filling: advanced ventricular contraction causes premature mitral valve closure and thereby premature ending of atrial contraction (A wave truncation). During LBBB, biventricular pacing increased $\mathrm{dP} / \mathrm{dt}_{\max }$ by $20 \%$; it was thereby almost restored to the baseline level during normal ventricular conduction.

Programming the device in this patient is a challenge. CRT is only effective during LBBB, and may have an adverse effect during normal intrinsic ventricular depolarisation. A rate-adaptive AV delay that is long at slow heart rates and shortens quickly when the heart rate increases to around $75 \mathrm{bpm}$ would promote predominantly intrinsic depolarisation during narrow QRS, and predominantly biventricular pacing during LBBB. However, most currently available CRT-D devices do not have this programming option. Since the heart rate was above the critical value during the majority of the time, one could argue that the CRT-D can be programmed according to usual practice in patients with permanent LBBB.

\section{Conclusions}

The presence of a rate-dependent LBBB demonstrated important basic CRT principles:

1. Induction of LBBB had a pronounced negative effect on cardiac function as reflected by an acute decrease in $\mathrm{LV} \mathrm{dP} / \mathrm{dt}_{\max }$;

2. During LBBB, biventricular pacing almost completely restored $\mathrm{LV} \mathrm{dP/dt} t_{\max }$ to baseline level during intrinsic normal ventricular conduction;

3. Biventricular pacing had no beneficial effect on LV dP/ $\mathrm{dt}_{\max }$ during normal ventricular conduction.

Funding This case was part of a study sponsored by University Medical Center Utrecht, Utrecht, the Netherlands, and which received financial support from Boston Scientific Corporation, St. Paul, MN, USA.

Open Access This article is distributed under the terms of the Creative Commons Attribution Noncommercial License which permits any noncommercial use, distribution, and reproduction in any medium, provided the original author(s) and source are credited.

\section{References}

1. Beshai JF, Grimm RA, Nagueh SF, Baker JH, Beau SL, Greenberg $\mathrm{SM}$, et al. Cardiac-resynchronization therapy in heart failure with narrow QRS complexes. N Engl J Med. 2007;357:2461-71.

2. Vardas PE, Auricchio A, Blanc JJ, Daubert JC, Drexler H, Ector H, et al. Guidelines for cardiac pacing and cardiac resynchronization therapy: the Task Force for Cardiac Pacing and Cardiac Resynchronization Therapy of the European Society of Cardiology. Developed in Collaboration with the European Heart Rhythm Association. Europace. 2007;9:959-98.

3. Nelson GS, Curry CW, Wyman BT, Kramer A, Declerck J, Talbot M, et al. Predictors of systolic augmentation from left ventricular preexcitation in patients with dilated cardiomyopathy and intraventricular conduction delay. Circulation. 2000;101:2703-9.

4. Mason DT. Usefulness and limitations of the rate of rise of intraventricular pressure (dp-dt) in the evaluation of myocardial contractility in man. Am J Cardiol. 1969;23:516-27.

5. Auricchio A, Stellbrink C, Sack S, Block M, Vogt Ju, Bakker P, et al. Long-term clinical effect of hemodynamically optimized cardiac resynchronization therapy in patients with heart failure and ventricular conduction delay. J Am Coll Cardiol. 2002;39:2026-33.

6. Vernooy K, Cornelussen RN, Verbeek XA, Vanagt WY, van Hunnik A, Kuiper $\mathrm{M}$, et al. Cardiac resynchronization therapy cures dyssynchronopathy in canine left bundle-branch block hearts. Eur Heart J. 2007;28:2148-55. 